\title{
Limits on Kaluza-Klein dark matter annihilation in the Sun from recent IceCube results
}

\author{
M. Colom i Bernadich, C. Pérez de los $\operatorname{Heros}^{\mathrm{a}}$ (i) \\ Department of Physics and Astronomy, Uppsala University, Box 516, 75120 Uppsala, Sweden
}

Received: 10 December 2019 / Accepted: 3 February 2020 / Published online: 15 February 2020

(C) The Author(s) 2020

\begin{abstract}
We interpret recent IceCube results on searches for dark matter accumulated in the Sun in terms of the lightest Kaluza-Klein excitation (assumed here to be the KaluzaKlein photon, $B^{1}$ ), obtaining improved limits on the annihilation rate in the Sun, the resulting neutrino flux at the Earth and on the $B^{1}$-proton cross-sections, for $B^{1}$ masses in the range $30-3000 \mathrm{GeV}$. These results improve previous results from IceCube in its 22-string configuration by up to an order of magnitude, depending on mass, but also extend the results to $B^{1}$ masses as low as $30 \mathrm{GeV}$.
\end{abstract}

\section{Introduction}

There are many astrophysical and cosmological observations that point to the existence of a dark matter component as a key constituent of the Universe. Constraints on the amount of baryons in the Universe from CMB measurements, from measurements of the abundance of primordial light elements and from searches for dark objects using microlensing have practically ruled out the possibility that dark matter consists of known Standard Model particles [1]. In the most popular picture, dark matter is composed by nonrelativistic Weakly Interacting Massive Particles (WIMPs) of yet unknown nature [2]. Among the many theories beyond the Standard Model of particle physics that predict new particles that could be viable dark matter candidates, Kaluza-Klein type models $[3,4]$ with universal extra dimensions (UED) ${ }^{1}$ provide a WIMP in the Kaluza-Klein photon $\left(B^{1}\right)$, the first excitation of the scalar gauge boson in the theory [5-10]. Usually denoted as the lightest Kaluza-Klein particle (LKP), it can have a mass in the range from a few hundred $\mathrm{GeV}$ (limit from rare decay processes $[11,12]$ ) to a few $\mathrm{TeV}$ (to avoid

\footnotetext{
${ }^{1}$ Universal in this context meaning that all the Standard Model fields are free to propagate also in the new dimension.
}

a e-mail: cph@physics.uu.se (corresponding author) overclosing the Universe), the mass being proportional to $1 / R$, where $R$ is the size of the extra dimension.

LKPs in the galactic halo will suffer the same fate as any other WIMP. Assuming a non-zero $B^{1}$-proton scattering cross section, $B^{1}$ 's in the galactic halo with Sun-crossing orbits can loose energy through interaction with the matter in the Sun and eventually sink into its core, where they would accumulate, thermalize, and annihilate into Standard Model particles [13-16], which in turn can lead to a detectable neutrino flux. Neutrino telescopes like AMANDA, IceCube, ANTARES and Baikal have searched for signatures of dark matter in this way, mainly focusing on the SUSY neutralino as WIMP candidate [17-20]. Additionally, both IceCube and ANTARES have set limits to the spin-dependent LKP-proton cross section [21-23]. The IceCube limits are based on the event selection and analysis searching for WIMP dark matter performed in Ref. [24].

In this letter we use the latest dark matter searches from the Sun by IceCube [18] to improve the limits on the KaluzaKlein photon cross section with protons. Given the increase in detector size ( 86 strings versus 22 in the previous IceCube analysis) and lifetime (104 days versus 532 days) the results presented in this letter improve those in Ref. [21] by up to an order of magnitude. Additionally, the presence of the lowenergy subdetector DeepCore allows to lower the explored LKP mass down to $30 \mathrm{GeV}$, compared to $250 \mathrm{GeV}$, the lowest mass studied in Ref. [21].

\section{LKP signatures from the Sun}

Table 1 shows the theoretical branching ratios of the $B^{1}$ selfannihilation processes in terms of the quark splitting mass:

$$
\Delta m=\frac{m_{q^{1}}-m_{B^{1}}}{m_{B^{1}}}
$$


Table 1 Table with the branching ratios of the main annihilation channels of $B^{1} B^{1} \rightarrow X \bar{X}$ in terms of two values of the quark splitting mass. The last channel corresponds to a Higgs-anti-Higgs pair. Source: Ref. [6]

\begin{tabular}{lll}
\hline Channel & $\Delta m=0$ & $\Delta m=0.14$ \\
\hline$v_{e} \bar{\nu}_{e}, \nu_{\mu} \bar{v}_{\mu}, \nu_{\tau} \bar{\nu}_{\tau}$ & 0.012 & 0.014 \\
$e^{+} e^{-}, \mu^{+} \mu^{-}, \tau^{+} \tau^{-}$ & 0.20 & 0.23 \\
$u \bar{u}, c \bar{c}, t \bar{t}$ & 0.11 & 0.077 \\
$d \bar{d}, s \bar{s}, b \bar{b}$ & 0.007 & 0.005 \\
$\phi \bar{\phi}$ & 0.023 & 0.027
\end{tabular}

where $m_{q^{1}}$ is the first fermion excitation in the Kaluza-Klein theory within universal extra dimensions [6]. The mass at which the LKP can be a good dark matter candidate depends on this splitting, which drives the co-annihilation with the higher KK modes which, in turn, determines the relic abundance [25]. Among the final annihilation products, only the weakly interacting neutrinos are able to escape from the Sun and be detected in neutrino observatories on Earth. Neutrinos resulting from these annihilations are also expected to be easily distinguishable from thermonuclear reaction products because the value of $B^{1}$ mass needed for it to be a good dark matter candidate $\left(m_{B^{1}}>\mathcal{O} 100 \mathrm{GeV}\right) .^{2}$

Assuming that the capture and annihilation rates of $B^{1}$ particles in the Sun, $\Gamma_{C}$ and $\Gamma_{A}$, have reached an equilibrium $[6,26]$, the relationship between them can be written as $\Gamma_{A}=$ $\frac{1}{2} \Gamma_{C}$, where the one half factor comes from the fact that one annihilation requires two captured $B^{1}$. This means that, assuming a particular velocity distribution for dark matter in the solar neighborhood and a solar structure model, the $B^{1}$ proton scattering cross section, which drives the capture, is proportional to the annihilation rate,

$\sigma^{i}=\lambda^{i}\left(m_{B^{1}}\right) \Gamma_{A}$

where the proportionality constant $\lambda^{i}\left(m_{B^{1}}\right)$ depends on the mass of the $B^{1}$, and the superscript $i$ can take the values SD (for spin-dependent cross section) or SI (for spinindependent cross section). The neutrinos produced in the core of the Sun have to be numerically propagated to a detector on Earth to predict the detectable neutrino flux per unit area and time in the detector, $\Phi_{v}$, which is proportional to the annihilation rate, $\Phi_{v}=\eta\left(m_{B^{1}}\right) \Gamma_{A}$. Such neutrino propagation must also take into account the solar composition, neutrino interaction processes with matter (such as absorption, re-emission, decays of secondary particles into neutrinos, etc.) and neutrino oscillations.

Note that the efficiency of LKP capture by the Sun is sensitive to the low-velocity tail of the, really unknown,

\footnotetext{
2 There are experimental limits on the lowest allowed mass for $B^{1}$ that we discuss below.
}

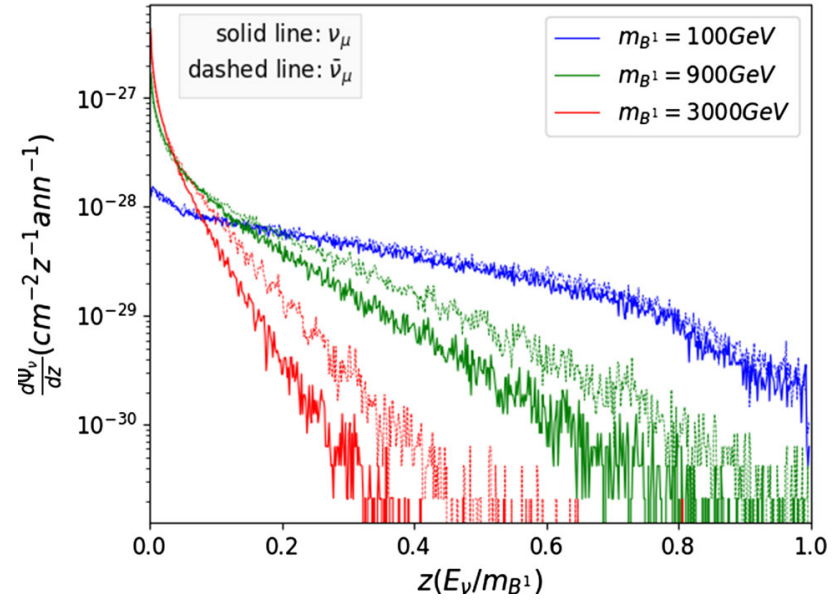

Fig. 1 Predicted energy spectra of muon neutrinos (solid lines) and anti-muon neutrinos (dashed lines) at the detector from $B^{1}$ annihilations, for three different values of $m_{B^{1}}$. An extra peak at the end of the spectra produced by neutrinos that escape the Sun without interacting has been omitted from the plot for the sake of legibility, but it is considered in the calculations. Note the normalization in relative energy units

LKP velocity distribution in the galaxy, $f(v)$ (lower velocity particles fall easier below the escape velocity of the Sun after collisions with solar matter). We assume here a standard Maxwellian dark matter velocity distribution. Although there is accumulating evidence for a non-Maxwellian component in $f(v)$ from recent Gaia and Sloan Digital Sky Survey (SDSS) data [28], the modifications to the Maxwellian distribution affect mainly the high-velocity tail, and are expected from simple kinematics to bear limited consequences for capture in the Sun. See Ref. [29] for a detailed discussion on the effects of astrophysical uncertainties on the calculation of dark matter capture in the Sun.

We simulated one million annihilation events at the core of the Sun and we propagated the neutrinos to a detector in the ice at latitude $90^{\circ} \mathrm{S}$ during the austral winter using WimpSim [27], for different assumed values of $m_{B^{1}}$. The simulations themselves do not include any information on the capture conditions or $\Gamma_{A}$, but they do require a solar structure model as an input for the propagation of neutrinos, in this case the one from Ref. [30]. Figure 1 shows the energy distribution of the muon (plus anti-muon) neutrino fluxes at the Earth per unit area $A$ and per annihilation in the Sun, as a function of reduced energy $z=E_{v} / m_{B^{1}}$,

$\frac{d \Psi_{v}}{d z}=\frac{d N_{v}}{d A d N_{A} d z}$

for some values of $m_{B^{1}}$. As expected, heavier $B^{1}$ particles produce steeper profiles in energy because of absorption of high-energy neutrinos on their way out of the Sun. The convolution of those spectra with the effective area of the detector gives a prediction of the number of events $\mu_{s}$ per number of annihilations $N_{A}$, expected at the detector 


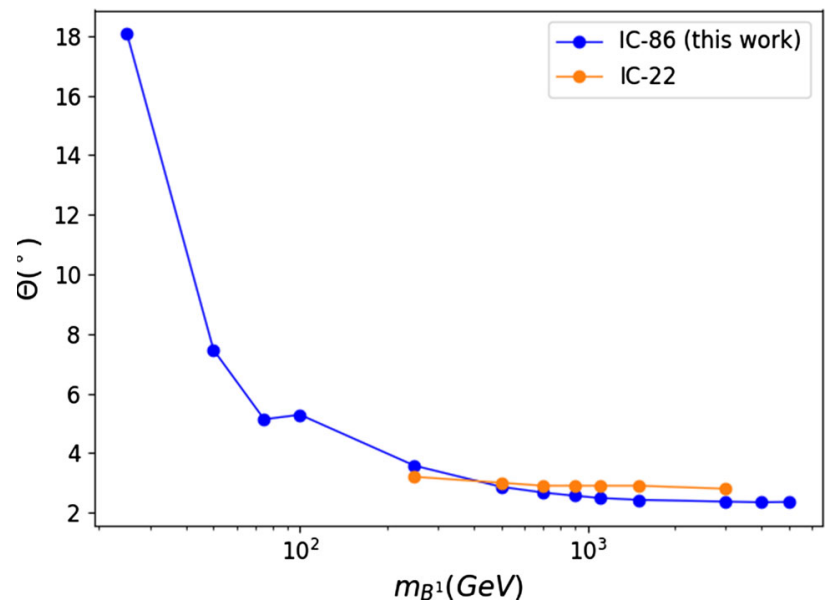

Fig. 2 Median angular resolution as a function of $B^{1}$ mass for the IceCube 86-string configuration (IC-86, blue line) and the 22-string configuration (IC-22, orange line). The bump at $m_{B^{1}}=100 \mathrm{GeV}$ in the former is due to the transition from DeepCore resolution to IceCube resolution [18]. Values calculated at the discrete mases denoted by the dots. Lines are a linear interpolation between the dots to guide the eye

$\frac{d \mu_{s}}{d N_{A}}=\int_{0}^{1} \frac{d \Psi_{v}}{d z} A_{e f f}(z) d z$

In order to take into account the finite angular resolution of the detector, the direction of each event has been randomly smeared using the median angular resolution of IceCube shown in Fig. 2, which has been computed from the median muon neutrino energy at every $m_{B^{1}}$ and the corresponding mean angular separation between the original neutrino and the reconstructed muon trajectories (see Ref. [18] for the explicit relation). $\Theta$ is used to spread the predicted signal (4) with a 2D Gaussian distribution centered around the Sun on the celestial sphere:

$\frac{d \mu_{s}}{d N_{A} d \psi}=\frac{d \mu_{s}}{d N_{A}} \frac{C}{\Theta^{2}} e^{-\psi^{2} / 2 \Theta^{2}} \sin \psi$

where $\psi$ is the angle from the Sun $\left(\psi_{\odot}=0\right)$, and $C$ a normalization constant. The expected angular distribution of the signal, for a few $B^{1}$ masses as a function of angle with respect to the Sun position is shown in Fig. 5.

\section{Data selection}

We use the muon-neutrino effective area corresponding to the latest solar dark matter search from IceCube [18], shown in Fig. 3. The figure shows the effective areas for IceCube and DeepCore for muon neutrinos as a function of neutrino energy, $E_{v}$, for the case of neutrinos arriving near the horizon, since the Sun is always close to the horizon in the South Pole. For this analysis, both curves are summed and the entire detector is treated as one single array. Only muon events are considered here since the long muon tracks allow pointing

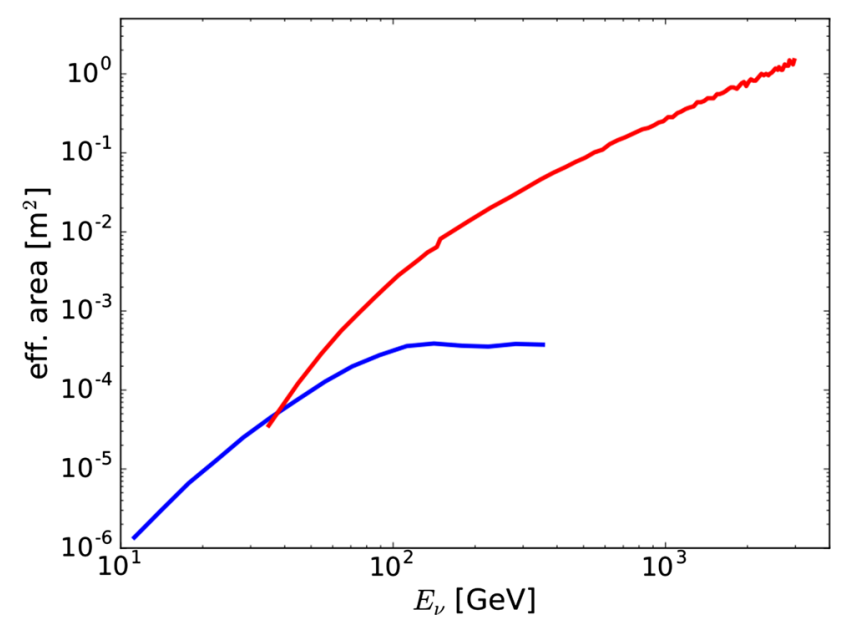

Fig. 3 The combined muon and anti-muon neutrino effective area for both the DeepCore (blue) and main IceCube array (red) for horizontally arriving neutrinos as a function of the neutrino energy. Uncertainties are not shown, but they can be as large as $30 \%$. Source: Ref. [18]

back to the Sun. Muon in what follows refer to both muons and anti-muons since IceCube can not distinguish between particles and antiparticles.

The predicted signal (Eq. 4) can be compared with the actual observed signal rate to compute $\Gamma_{A}$ :

$\frac{d \mu_{s}}{d t}=\frac{d N_{A}}{d t} \frac{d \mu_{s}}{d N_{A}}=\Gamma_{A} \frac{d \mu_{s}}{d N_{A}}$

and $\Gamma_{A}$ is used to calculate the $B^{1}$-proton scattering cross sections through Eq. (2). The conversion factors $\lambda^{i}\left(m_{B^{1}}\right)$ are obtained with the DarkSUSY software [31], and include the information on the solar structure and the dark matter velocity distribution in the solar neighborhood [26]. Figure 4 shows the expected number of events at the detector per annihilation as a function of $B^{1}$ mass calculated with Eq. (4)

We use the data from Fig. 6 in Ref. [18], which we present here combined for DeepCore and IceCube in Fig. 5, in order to extract limits on the spin-dependent $B^{1}$-proton cross section as a function of $B^{1}$ mass. The data set was obtained during 532 days of exposure between May 2011 and May 2014. As thoroughly explained in Ref. [18], several filters were applied to the data sample in order to minimize the presence of background. Data was only taken into account if measured during austral winters, when the Sun is below the horizon, in order to avoid overlap with atmospheric muons originating from cosmic-ray induced showers in the atmosphere above the detector. For this reason, only muons with upward trajectories were selected. Still, atmospheric mиоn neutrinos created at any declination can cross the Earth without interacting and reach the detector from below, being an irreducible background. Boosted decision trees were used to maximize signal separation and reduce background. 


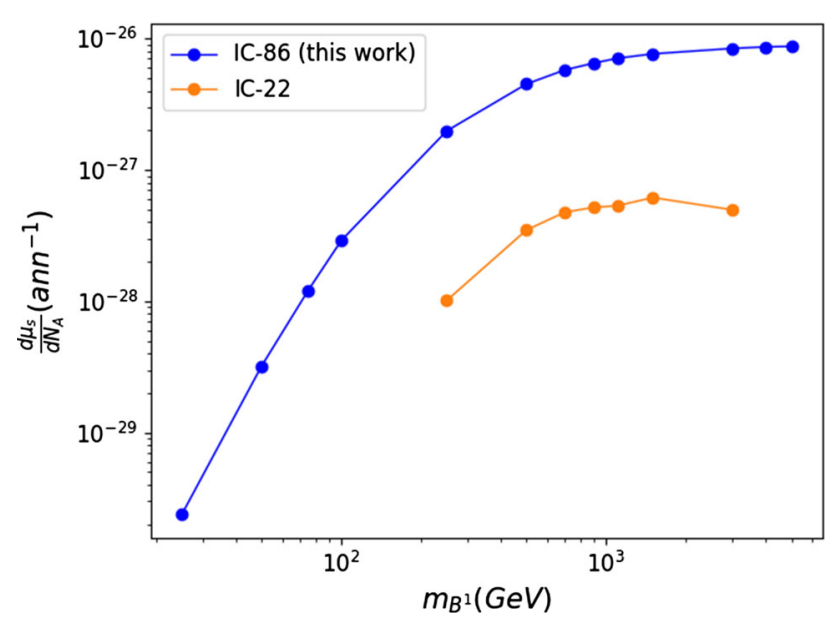

Fig. 4 Expected number of events in IceCube per annihilation as a function of $B^{1}$ mass. The blue dots (IC-86) show the result of this work (using Eq. (4) with the effective area of Ref. [18]), while the orange dots show the result using the effective area of the 22-string IceCube configuration (IC-22) [24], which was the basis for the previous KaluzaKlein analysis [21]. Values calculated at the discrete mases denoted by the dots. Lines are just a linear interpolation between the dots to guide the eye

\section{Results}

Figure 5 shows that no statistically significant deviation from the expected background was detected in the IceCube solar analysis, so a $90 \%$ confidence level limit on the $B^{1}$-proton cross section can be extracted from the data by setting a limit to $\Gamma_{A}$. The amount of expected signal events per unit of time can be estimated as

$$
\frac{d \mu_{s}}{d t} \simeq \frac{\mu_{s}}{\tau}
$$

where $\tau$ is the total exposure time, 532 days in our case. We proceed with a "counting experiment", comparing the number of background events to the number of observed events extracted from Fig. 5, and construct Poisson confidence intervals for the signal strength $\mu_{s}$, calculated with the Neyman method using the algorithm developed in Ref. [32], including the detector systematic uncertainties from Ref. [18]. A limit on $\mu_{s}$ is easily translated to a limit on $\Gamma_{A}$ through equation (6) and further to limits on $\sigma^{S I}$ and $\sigma^{S D}$ through equation (2). Table 2 shows the results for a series of $B^{1}$ masses. Limits at $90 \%$ confidence level on the signal strength $\left(\mu_{S}\right)$, the annihilation rate in the Sun $\left(\Gamma_{A}\right)$, the muon flux at the detector above $1 \mathrm{GeV}\left(\Phi_{\mu}\right)$ and the spin-independent and spin-dependent cross sections $\left(\sigma^{S I}\right.$ and $\sigma^{S D}$ ) are shown, along with the median angular resolution $\left(\Delta \theta_{\nu}\right)$ and mean muon energy at the detector $\left(\left\langle E_{\mu}\right\rangle\right)$ for each signal model.

Figure 6 shows $\sigma^{S D}$ versus $B^{1}$ mass for the current analysis (blue dots) and the previously published analysis by IceCube in the 22-string configuration (orange dots), where it

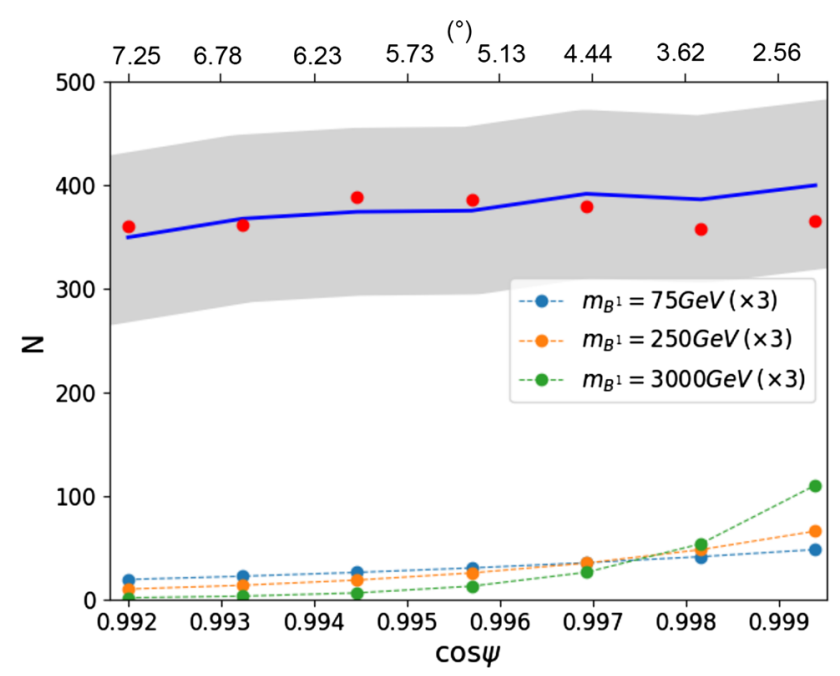

Fig. 5 Expected background (dark blue line) within the range of maximum systematic uncertainties (shaded area), the actual number of detected events at every angular bin (red dots) and the expected signal (multiplied by three for visualization) for some values of $m_{B^{1}}$ (light blue, orange and green), as a function of angle with respect to the Sun (in cosine in the lower axis and degrees in the upper). Data from Ref. [18]

can be seen that the constraints have been improved by up to one order of magnitude. The figure also shows the results from the ANTARES collaboration [23] (black curve). The shaded area shows the disfavoured mass region for the first Kaluza-Klein excitation obtained from searches for UED at the LHC [33], where a limit on $1 / \mathrm{R}(\mathrm{GeV})$ is obtained by combining several searches for events with large missing transverse momentum or monojets by ATLAS and CMS at $8 \mathrm{TeV}$ and $13 \mathrm{TeV}$ center of mass energies. Collider searches provide a complementary approach to indirect searches for dark matter in the form of Kaluza-Klein modes with neutrino telescopes, being competitive in different regions of the LKP mass range. Additional constraints from cosmology (that the $B^{1}$ must have a relic density compatible with the estimated dark matter density from CMB measurements) require the mass of the $B^{1}$ to be below $\sim 1.6 \mathrm{TeV}[9,25,35]$. Thus, taken all results together, the allowed parameter space for the $B^{1}$ to constitute the only component of dark matter in the Universe is currently quite restricted, but non-minimal UED models, not probed here, can still provide viable dark matter candidates [10].

From the experimental point of view, a few simplifying assumptions have been made to obtain the presented results. Uncertainties on the solar structure model and dark matter velocity distribution have been ignored, and binned data (from Fig. 5) have been used instead of a continuous sample of individual events, which limits the statistical power of the analysis. Even with these approximations, the IceCube limit presented in this letter consid- 
Table 2 90\% confidence level upper limits on the signal, the annihilation rate in the Sun, the muon flux at the detector above $1 \mathrm{GeV}$ and the spin independent and spin dependent $B^{1}$-proton cross sections for several values of the $B^{1}$ mass. The last two columns show the median angular resolution and the muon mean energy at the detector

\begin{tabular}{llllllll}
\hline$m_{B^{1}}(\mathrm{GeV})$ & $\mu_{S}$ & $\Gamma_{A}(\mathrm{ann} \mathrm{s})$ & $\Phi_{\mu}\left(\mathrm{km}^{-2} \mathrm{year}^{-2}\right)$ & $\sigma^{S I}\left(\mathrm{~cm}^{2}\right)$ & $\sigma^{S D}\left(\mathrm{~cm}^{2}\right)$ & $\Delta \theta_{\nu}\left({ }^{\circ}\right)$ & $\left\langle E_{\mu}\right\rangle(\mathrm{GeV})$ \\
\hline 25 & 907.6 & $8.3 \times 10^{24}$ & $2.0 \times 10^{4}$ & $5.1 \times 10^{-41}$ & $6.0 \times 10^{-39}$ & \pm 18.1 & 6.8 \\
50 & 186.7 & $1.3 \times 10^{23}$ & $1.1 \times 10^{3}$ & $1.4 \times 10^{-42}$ & $2.8 \times 10^{-40}$ & \pm 7.5 & 13.3 \\
75 & 111.8 & $2.0 \times 10^{22}$ & $3.5 \times 10^{2}$ & $3.4 \times 10^{-43}$ & $9.3 \times 10^{-41}$ & \pm 5.1 & 19.3 \\
100 & 114.0 & $8.6 \times 10^{21}$ & $2.5 \times 10^{2}$ & $2.0 \times 10^{-43}$ & $6.7 \times 10^{-41}$ & \pm 5.3 & 25.8 \\
250 & 80.5 & $8.9 \times 10^{20}$ & 98 & $7.1 \times 10^{-44}$ & $4.0 \times 10^{-41}$ & \pm 3.6 & 54.7 \\
500 & 73.1 & $3.5 \times 10^{20}$ & 76 & $8.3 \times 10^{-44}$ & $6.3 \times 10^{-41}$ & \pm 2.9 & 88.0 \\
700 & 72.2 & $2.7 \times 10^{20}$ & 73 & $1.1 \times 10^{-43}$ & $9.4 \times 10^{-41}$ & \pm 2.7 & 107.6 \\
900 & 71.8 & $2.4 \times 10^{20}$ & 71 & $1.6 \times 10^{-43}$ & $1.4 \times 10^{-40}$ & \pm 2.6 & 117.1 \\
1100 & 71.5 & $2.2 \times 10^{20}$ & 71 & $2.1 \times 10^{-43}$ & $1.9 \times 10^{-40}$ & \pm 2.5 & 126.9 \\
1500 & 71.4 & $2.0 \times 10^{20}$ & 71 & $3.4 \times 10^{-43}$ & $3.2 \times 10^{-40}$ & \pm 2.4 & 135.1 \\
3000 & 71.3 & $1.8 \times 10^{20}$ & 70 & $1.2 \times 10^{-42}$ & $1.1 \times 10^{-39}$ & \pm 2.4 & 148.3 \\
4000 & 71.2 & $1.8 \times 10^{20}$ & 70 & $2.0 \times 10^{-42}$ & $2.0 \times 10^{-39}$ & \pm 2.3 & 150.3 \\
5000 & 71.2 & $1.8 \times 10^{20}$ & 71 & $3.1 \times 10^{-42}$ & $3.1 \times 10^{-39}$ & \pm 2.4 & 151.5
\end{tabular}

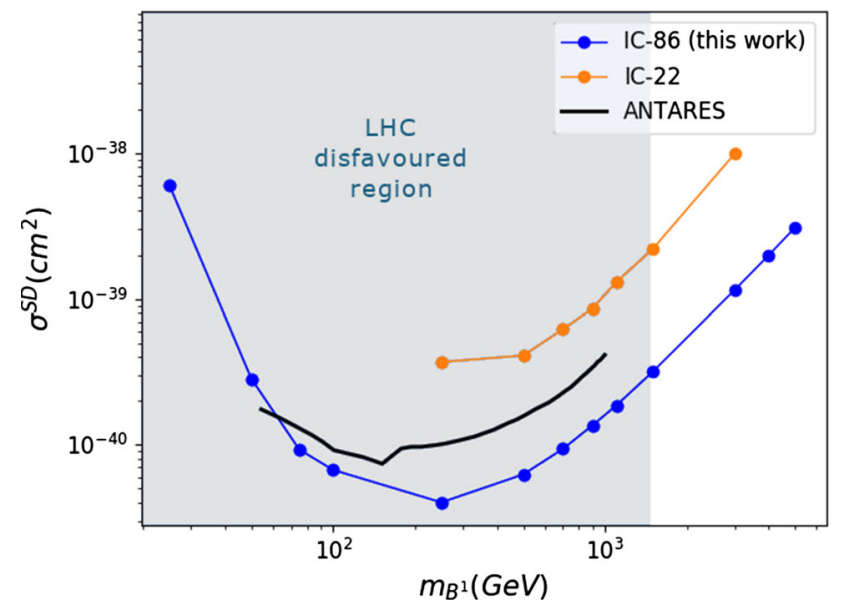

Fig. $690 \%$ confidence level upper limit on the spin-dependent $B^{1}$ proton scattering cross section as a function of $B^{1}$ mass. Blue curve (IC-86): this work. Orange curve (IC-22): previous IceCube limit on LKP cross section from the 22-string IceCube configuration [21]. Black line: limits from ANTARES [23]. Limits from IceCube have been evaluated at the discrete masses denoted by the dots. Lines are just a linear interpolation between the dots to guide the eye. The shaded area represents the disfavoured region (at 95\% confidence level) on the mass of the LKP from the LHC [33]

erably improves over those published in Refs. [21] and [23].

Data Availability Statement This manuscript has no associated data or the data will not be deposited. [Authors' comment: This article is based on data presented in Ref. [19].]

Open Access This article is licensed under a Creative Commons Attribution 4.0 International License, which permits use, sharing, adaptation, distribution and reproduction in any medium or format, as long as you give appropriate credit to the original author(s) and the source, pro- vide a link to the Creative Commons licence, and indicate if changes were made. The images or other third party material in this article are included in the article's Creative Commons licence, unless indicated otherwise in a credit line to the material. If material is not included in the article's Creative Commons licence and your intended use is not permitted by statutory regulation or exceeds the permitted use, you will need to obtain permission directly from the copyright holder. To view a copy of this licence, visit http://creativecomm ons.org/licenses/by/4.0/.

Funded by SCOAP ${ }^{3}$.

\section{References}

1. K. Garrett, G. Duda, Dark matter: a primer. Adv. Astron. 2011, $968283(2011)$

2. G. Bertone, D. Hooper, J. Silk, Particle dark matter: evidence, candidates and constraints. Phys. Rep. 405, 279 (2005)

3. T. Kaluza, Zum Unittatsproblem der Physik. Sitzungsber. Preuss. Akad. Wiss. Berlin (Math. Phys.) 1921, 966 (1921) [Int. J. Mod. Phys. D 27(14), 1870001 (2018)]

4. O. Klein, Quantum theory and five-dimensional theory of relativity. Z. Phys. 37, 895 (1926)

5. H.C. Cheng, J.L. Feng, K.T. Matchev, Kaluza-Klein dark matter. Phys. Rev. Lett. 89, 211301 (2002)

6. D. Hooper, G.D. Kribs, Probing Kaluza-Klein dark matter with neutrino telescopes. Phys. Rev. D 67, 055003 (2003)

7. G. Servant, T.M.P. Tait, Is the lightest Kaluza-Klein particle a viable dark matter candidate? Nucl. Phys. B 650, 391 (2003)

8. D. Hooper, S. Profumo, Dark matter and collider phenomenology of universal extra dimensions. Phys. Rep. 453, 29 (2007)

9. M. Blennow, H. Melbeus, T. Ohlsson, Neutrinos from KaluzaKlein dark matter in the Sun. JCAP 1001, 018 (2010)

10. T. Flacke, D.W. Kang, K. Kong, G. Mohlabeng, S.C. Park, Electroweak Kaluza-Klein dark matter. JHEP 1704, 041 (2017)

11. A. Freitas, U. Haisch, Anti-B $\rightarrow X(\mathrm{~s})$ gamma in two universal extra dimensions. Phys. Rev. D 77, 093008 (2008)

12. U. Haisch, A. Weiler, Bound on minimal universal extra dimensions from anti-B $\rightarrow$ X(s)gamma. Phys. Rev. D 76, 034014 (2007) 
13. D.N. Spergel, W.H. Press, Effect of hypothetical, weakly interacting, massive particles on energy transport in the solar interior. Astrophys. J. 294, 663 (1985)

14. W.H. Press, D.N. Spergel, Capture by the sun of a galactic population of weakly interacting massive particles. Astrophys. J. 296, 679 (1985)

15. T.K. Gaisser, G. Steigman, S. Tilav, Limits on cold dark matter candidates from deep underground detectors. Phys. Rev. D 34, 2206 (1986)

16. J.S. Hagelin, K.W. Ng, K.A. Olive, A high-energy neutrino signature from supersymmetric relics. Phys. Lett. B 180, 375 (1986)

17. R. Abbasi et al., Multi-year search for dark matter annihilations in the Sun with the AMANDA-II and IceCube detectors. Phys. Rev. D 85, 042002 (2012)

18. M.G. Aartsen et al., Search for annihilating dark matter in the Sun with 3 years of IceCube data. Eur. Phys. J. C 77(3), 146 (2017). (Erratum: [Eur. Phys. J. C 79 no.3, 214, (2019)])

19. S. Adrian-Martinez et al., Limits on dark matter annihilation in the Sun using the ANTARES neutrino telescope. Phys. Lett. B 759, 69 (2016)

20. A.D. Avrorin et al., Search for neutrino emission from relic dark matter in the Sun with the Baikal NT200 detector. Astropart. Phys. 62, 12 (2015)

21. R. Abbasi et al., Limits on a muon flux from Kaluza-Klein dark matter annihilations in the Sun from the IceCube 22-string detector. Phys. Rev. D 81, 057101 (2010)

22. O. Engdegård, $A$ search for dark matter in the Sun with AMANDA and IceCube. Ph.D. thesis, Uppsala University, Acta Universitatis Upsaliensis 2011. ISBN: 978-91554-8218-3. http://www.diva-portal.org/smash/get/diva2:453245/ FULLTEXT01.pdf. Accessed 13 Jan 2020

23. J.D. Zornoza, Search for dark matter in the Sun with the ANTARES neutrino telescope in the CMSSM and mUED frameworks. Nucl. Instrum. Methods A 725, 76 (2013)
24. R. Abbasi et al., Limits on a muon flux from neutralino annihilations in the Sun with the IceCube 22-string detector. Phys. Rev. Lett. 102, 201302 (2009)

25. G. Belanger, M. Kakizaki, A. Pukhov, Dark matter in UED: the role of the second KK level. JCAP 1102, 009 (2011)

26. G. Wikström, J. Edsjö, Limits on the WIMP-nucleon scattering cross-section from neutrino telescopes. JCAP 0904, 009 (2009)

27. J. Edsjö, J. Elevant, C. Niblaeus, WimpSim Neutrino Monte Carlo. http://wimpsim.astroparticle.se. Accessed 15 Feb 2019

28. L. Necib, M. Lisanti, V. Belokurov, Inferred evidence for dark matter kinematic substructure with SDSS-Gaia. Astrophys. J. 874, 3 (2019)

29. M. Danninger, C. Rott, Solar WIMPs unravelled: experiments, astrophysical uncertainties, and interactive tools. Phys. Dark Univ. 5-6, 35 (2014)

30. A. Serenelli, S. Basu, J.W. Ferguson, M. Asplund, New solar composition: the problem with solar models revisited. Astrophys. J. 705, L123 (2009)

31. T. Bringmann, J. Edsjö, P. Gondolo, P. Ullio, L. Bergström, DarkSUSY 6: an advanced tool to compute dark matter properties numerically. JCAP 1807, 033 (2018)

32. J. Conrad, O. Botner, A. Hallgren, C. Pérez de los Heros, Including systematic uncertainties in confidence interval construction for Poisson statistics. Phys. Rev. D 67, 012002 (2003)

33. N. Deutschmann, T. Flacke, J.S. Kim, Current LHC constraints on minimal universal extra dimensions. Phys. Lett. B 771, 515 (2017)

34. J. Beuria, A. Datta, D. Debnath, K.T. Matchev, LHC collider phenomenology of minimal universal extra dimensions. Comput. Phys. Commun. 226, 187 (2018)

35. S. Arrenberg, L. Baudis, K. Kong, K.T. Matchev, J. Yoo, KaluzaKlein dark matter: direct detection vis-a-vis LHC. PoS IDM 2008, 059 (2008) 